\title{
L'impact du commerce bio-équitable sur la santé des producteurs marginalisés au Kenya
}

\author{
Leonardo Becchetti ${ }^{1}$ \\ Giuseppina Gianfreda ${ }^{2}$ \\ 1 Université Roma Tor Vergata \\ Faculté d'économie \\ Via Columbia 2 \\ 00133 Rome \\ Italie \\ $<$ becchetti@economia.uniroma2.it> \\ 2 Université de Viterbe \\ Faculté de sciences politiques \\ Via San Carlo, 32 \\ 01100 Viterbo \\ <ggianfreda@unitus.it>
}

\begin{abstract}
Résumé
Les consommateurs responsables d'Europe et des États-Unis sont de plus en plus disposés à payer une "prime éthique " pour rémunérer la valeur sociale et environnementale des produits issus du commerce équitable. Un des critères du commerce équitable (dont l'objectif est d'améliorer le bien-être et de renforcer les capacités des producteurs marginalisés) concerne la santé des producteurs et la création de conditions de travail saines. Nous évaluons la pertinence de ce critère en comparant les nombres de jours perdus pour cause de maladie par des producteurs kenyans affiliés ou non au commerce équitable. Nous montrons que le nombre d'années d'affiliation au commerce équitable joue un rôle significatif sur le nombre de jours perdus pour cause de maladie.
\end{abstract}

Mots clés : commerce équitable ; santé ; évalution de l'impact.

Thèmes : économie et développement rural ; productions végétales ; systèmes agraires.

\section{Abstract \\ Does Consumption Heal Producers? The Effect of Fair Trade on Marginalised Producers' Health in Kenya}

Concerned consumers in the US and Europe are increasingly willing to pay an "ethical premium" for the social and environmental value of fair trade products. One of the fair trade criteria (aimed to enhance wellbeing and capacity building of marginalised producers) relates to producers health and creation of healthy working conditions. We evaluate its significance by comparing days lost for illness of FT and non FT affiliated Kenyan farmers. We find that FT affiliation years have a significant effect in the expected direction on the dependent variable.

Key words: fair trade; health; impact assessment.

Subjects: economy and rural development; farming systems; vegetal productions. es produits issus du commerce équitable proviennent de chaînes de valeur innovatrices, ayant pour but de générer une valeur économique plus élevée et de procurer des bénéfices sociaux aux producteurs marginalisés. Le dispositif du commerce équitable comprend :

- un renforcement des capacités ;

- une prime de prix contracyclique permettant de compenser le faible pouvoir de marché des producteurs et dont le niveau ne peut être inférieur à un "seuil de subsistance " prédéfini (en Équateur, le prix sur le marché conventionnel d'une caisse de 1,14 kg de bananes était de 2,91 dollars US en 2005, contre un prix du commerce équitable de 7,75 dollars US). D'autres données concernant ces primes pour les prix du café et du cacao au cours des 20 dernières années sont disponibles auprès des auteurs ;

- un mécanisme de préfinancement dont le but est de casser le pouvoir monopolistique des prêteurs locaux. Une partie de la prime est versée à l'association de producteurs locale pour financer l'offre de biens publics locaux (formations, installations sanitaires, accompagnement éducatif etc.).

Plusieurs enquêtes menées dans la plupart des pays industrialisés révèlent un consentement à payer élevé des 
consommateurs pour les attributs sociaux et environnementaux des biens de consommation (Becchetti et Rosati, 2007). Les préférences révélées confirment que les choix virtuels se traduisent par des achats réels, d'une part parce que les consommateurs paient une prime pour ces éléments immatériels intégrés dans des produits, et, d'autre part, parce que la part de marché des produits issus du commerce équitable a atteint un niveau remarquable sur certains segments (47\% pour les bananes en Suisse, 20 \% du café moulu au Royaume-Uni, soit une croissance annuelle des ventes nettes globales de $20 \%$ au cours des cinq dernières années). Maseland et De Vaal (2002) proposent une évaluation théorique des effets du commerce équitable au regard des théories de commerce international. S'appuyant sur la théorie traditionnelle du commerce international, ces auteurs expliquent dans quelles circonstances les subventions implicites du commerce équitable augmentent le bien-être. D'autres articles traitent de manière pertinente différents aspects de l'impact du commerce équitable: ainsi Moore (2004), Hayes (2004) et Redfern et Sneker (2002) soulignent qu'il est impossible d'analyser le commerce équitable de façon statique en tant que distorsion du prix de marché et qu'il faut l'évaluer de façon dynamique, en tenant compte de son effet potentiellement positif sur le renforcement des capacités des producteurs et sur l'accès au marché de ces derniers. À l'heure actuelle, la littérature empirique dans ce domaine se limite à des études de cas et à quelques analyses économétriques. Bacon (2005) montre ainsi, sans toutefois contrôler l'effet d'autres facteurs explicatifs, que, pour un échantillon de producteurs de café du Guatemala, l'accès aux marchés certifiés produit un effet positif sur le prix de vente. Pariente (2000) observe un impact positif du prix minimum sur la stabilité du revenu des producteurs de café - qui diminue ainsi la probabilité qu'ils ont de tomber sous le seuil de pauvreté - au sein de la coopérative Coocafé au Costa Rica. Becchetti et Costantino (2008) montrent, après avoir contrôlé le biais de sélection, que l'affiliation au commerce équitable exerce un impact significatif sur plusieurs indicateurs de bien-être, même si cet élément ne semble pas améliorer de façon significative l'investissement en capital humain. Dans le cadre d'une enquête parallèle menée sur deux coopératives de producteurs au Pérou, Becchetti,
Costantino et Portale (2007) mettent en avant deux types d'externalités: les acteurs affiliés au commerce équitable jouissent d'un pouvoir de négociation plus important auprès des intermédiaires locaux et, dans un des deux projets, le commerce équitable permet d'améliorer la situation des acteurs locaux non affiliés au commerce équitable. La prime de prix du commerce équitable (différence entre le prix du commerce équitable et le prix offert par les importateurs traditionnels) dépasse les $100 \%$ dans cette étude. Les seuls à payer davantage que les importateurs du commerce équitable sont les "voyageurs éthiques" ". Dans les deux projets, le revenu des producteurs, l'investissement en capital humain, les dépenses hebdomadaires d'alimentation, la part de la consommation non alimentaire dans les dépenses totales, le niveau de vie relatif autoévalué et l'estime de soi profession. nelle sont corrélés de façon significative et positive avec le nombre d'années d'affiliation au commerce équitable.

Notre article examine l'impact de l'affiliation au commerce équitable sur une dimension jusqu'à présent inexplorée: la santé des travailleurs. Ce critère est doublement important. Primo, il est bien connu que la santé est l'une des dimensions les plus importantes du bien-être individuel et du sentiment de satisfaction globale de la vie (Frey et Stutzer, 2002a, 2002b; Clark et al., 2006). Secundo, la santé influence de façon cruciale la productivité individuelle et peut être considérée comme un facteur indirect de convergence conditionnelle dans les modèles de croissance, dans la mesure où de mauvaises conditions sanitaires pénalisent l'investissement dans l'éducation et réduisent le nombre d'heures travaillées. Des études empiriques ont souligné une relation de double causalité entre l'état de santé et les conditions socio-économiques. D'une part, on considère qu'une mauvaise santé influence la capacité à constituer un revenu et à accumuler de la richesse, car elle affecte la productivité et les salaires et accroît les dépenses de santé. D'autre part, un

\footnotetext{
1 Le tourisme éthique et responsable est une initiative globale dont le but est d'organiser des activités touristiques pour minimiser l'impact des voyageurs sur les pays visités et nourrir les échanges socioculturels avec la population rencontrée. En général, l'élément de responsabilité sociale implique qu'une part plus importante de la valeur générée par ce genre de tourisme revienne à la population locale.
}

revenu limité suppose du stress physique et un accès limité aux soins. La relation entre les revenus et la santé se traduit de façon très différente dans les pays riches et les pays pauvres, car les pays pauvres souffrent tout particulièrement de problèmes comme la malnutrition, les mauvaises conditions sanitaires et l'analphabétisme. Les canaux par lesquels une plus grande richesse influence la santé dans les pays pauvres ne se limitent pas à un meilleur accès aux soins et à la disponibilité en médicaments. Il n'est pas surprenant que les études empiriques soulignent l'importance de facteurs tels que la qualité nutritionnelle, l'accès à l'eau propre et à l'électricité, et toutes les infrastructures favorisant une meilleure hygiène. Il convient également de noter que l'hygiène est étroitement liée à l'éducation dans les pays en voie de développement. Jusqu'à présent, les travaux se sont surtout concentrés sur la relation entre l'éducation et la santé des adultes, ainsi que sur la relation entre l'éducation des parents et la santé des enfants. Pour une vue d'ensemble de cette littérature concernant les pays pauvres, voir Deaton (2006) et Strauss et Thomas (1998).

Parmi les critères établis par WFTO (World Fair Trade Organisation), la fédération internationale du commerce équitable regroupant des importateurs et des associations de producteurs), figurent les " conditions de travail" définies comme un environnement de travail sain pour les producteurs. Plusieurs éléments des accords sur le commerce équitable contribuent à la réalisation de cet objectif. Des avantages tels que la stabilisation des prix, l'octroi d'une prime de prix contracyclique et le développement d'une capacité intégrée permettent de réduire les risques liés à l'activité économique. Becchetti et Costantino (2008) montrent que la réduction des risques est liée de manière significative à la réduction de l'épargne de précaution, à une hausse de la consommation et des dépenses sanitaires, et à l'amélioration de la qualité nutritionnelle. On s'attend à ce que ces facteurs aient un effet positif significatif sur la santé. Le soutien au développement des techniques de l'agriculture biologique peut également contribuer à réduire l'exposition des agriculteurs aux maladies, de façon directe - en diminuant l'usage des produits chimiques - et indirecte - dans les pays pauvres, une part importante de la production est généralement autoconsommée. Cependant, il convient de noter que la question des 
avantages de l'agriculture biologique par rapport à l'agriculture conventionnelle reste un sujet de controverses. Par exemple, même si un moindre recours aux pesticides peut avoir des effets bénéfiques sur la santé, les produits alimentaires conventionnels restent moins chers et un accroissement des quantités consommées peut également améliorer la santé des populations. Trewavas (2004) discute cette question dans le cas du RoyaumeUni. De même, alors que la littérature (Worthington, 2001) souligne l'existence de différences nutritionnelles entre les aliments issus de l'agriculture biologique et les aliments conventionnels, l'on ne dispose toujours pas de preuves pour soutenir ou réfuter l'hypothèse d'une meilleure qualité nutritionnelle de l'alimentation biologique par rapport à l'alimentation conventionnelle (Williams, 2002).

\section{Le projet " Meru Herbs »}

Meru Herbs est une société commerciale fondée en 1991 par une association d'agriculteurs locaux (le comité des eaux de Ng'uuru Gakirwe) pour collecter des fonds via la commercialisation des produits alimentaires, financer l'aménagement hydraulique du fleuve Kitcheno et ainsi approvisionner les foyers et fermes en eau. Le Comité a été fondé par 430 familles occupant des parcelles (de 4 à 16 hectares) octroyées par le gouvernement kenyan pendant les années 1960. Les parcelles se situent dans les districts de Meru Central et de Tharaka, à 200 kilomètres de Naïrobi sur le versant Est du Mont Kenya. Dès sa création, Meru Herbs a développé un partenariat expérimental avec CTM (premier importateur italien de produits issus du commerce équitable) dans le but de briser le monopsone des commerçants de Naïrobi pour la commercialisation des produits alimentaires. En 2000, Meru Herbs s'est vu délivrer une certification biologique par l'entreprise britannique Soil Association Certification Ltd. Aujourd'hui, jusqu'à $97 \%$ du chiffre d'affaires net de Meru Herbs est réalisé avec des organisations de commerce équitable. Les relations commerciales des agriculteurs locaux avec Meru Herbs ne sont pas exclusives : les agriculteurs continuent à vendre localement jusqu'à $40 \%$ de leur production.
Pour s'affilier à Meru Herbs, les agriculteurs ont dû obtenir la certification biologique (ou doivent avoir entamé la procédure pour l'obtenir). Meru Herbs achète également les fruits d'agriculteurs conventionnels pour la production de confitures. Il est ainsi possible de regrouper les agriculteurs qui font du commerce avec Meru Herbs en trois catégories : les membres à 100 \%, c'est-à-dire des agriculteurs biologiques ayant signé un contrat avec Meru Herbs ("agriculteurs biologiques ") ; les agriculteurs ayant récemment signé un contrat avec Meru Herbs et qui ont entamé la procédure d'obtention de certification biologique ("agriculteurs en conversion ") ; et de simples vendeurs de fruits à l'association de producteurs ("fruticulteurs"). Pour souligner l'effet du projet commerce équitable-Meru Herbs sur les variables socio-économiques, l'enquête comprend un "groupe témoin ", sélectionné parmi les agriculteurs non affiliés vivant dans la même région. Pour mener notre analyse, nous avons sélectionné de manière aléatoire des nombres égaux d'individus dans les quatre groupes mentionnés ci-dessus (biologique, conversion, fruticulteurs, témoin) parmi la population de 474 agriculteurs vivant dans la région du projet des eaux de Ng'uuru Gakirwe. Comme Meru Herbs a été dès son origine lié aux organismes du commerce équitable, il est impossible de distinguer les bénéfices associés à l'affiliation auprès de Meru Herbs de ceux découlant des relations avec le commerce équitable.

En janvier 2005, les membres des quatre groupes ont répondu, au cours d'entretiens individuels, à une enquête comprenant 100 questions. Les informations obtenues concernaient la démographie, les conditions de vente des produits, les sources de revenu monétaire et non monétaire, la santé, les dépenses de consommation alimentaire et la qualité nutritionnelle, le nombre d'années d'éducation et la situa- tion professionnelle des membres du ménage, des indicateurs sociaux et d'aptitude, des mesures subjectives de la satisfaction concernant les prix et les conditions de vie ainsi que des indicateurs du capital social.

\section{Résultats descriptifs}

Nous mesurons l'état de santé par le nombre de jours de travail perdus pour cause de maladie. Notre questionnaire indique quatre catégories de réponses des agriculteurs : des individus n'ayant perdu aucun jour de travail pour cause de maladie, des individus ayant perdu entre 1 et 5 jours, des individus ayant perdu entre 6 et 15 , et des individus ayant perdu plus de 15 jours. Le tableau 1 montre que les agriculteurs du groupe " agriculteurs biologiques " sont les individus les plus représentés dans le groupe des agriculteurs n'ayant perdu aucun jour de travail et les moins représentés parmi les agriculteurs ayant perdu plus de 15 jours. Leur présence dans chacune des catégories diminue au fur et à mesure que le nombre de jours perdus augmente. Aucun autre groupe ne démontre une distribution aussi particulière que le groupe "agriculteurs biologiques ". Ce résultat est d'autant plus significatif que les agriculteurs biologiques affichent une moins bonne performance par rapport aux facteurs identifiés dans la littérature comme des déterminants majeurs de la santé (éducation et âge).

Les caractéristiques sommaires des quatre groupes sont présentées dans le tableau 2. Nous indiquons également (entre crochets) des intervalles de confiance à $95 \%$ pour les valeurs moyennes du groupe présentées dans le tableau. Quand les intervalles de confiance ne se chevauchent pas, la différence des moyennes entre deux valeurs de groupes données est statistiquement

\section{Tableau 1. La performance des quatre groupes en termes de jours de travail perdus pour cause de maladie.}

Table 1. Performance of the four groups in terms of days lost for illness.

\begin{tabular}{lcccc}
\hline & $\begin{array}{c}\mathbf{0} \text { jour } \\
\text { perdu }\end{array}$ & $\begin{array}{c}\mathbf{1} \text { à } \mathbf{5} \text { jours } \\
\text { perdus }\end{array}$ & $\begin{array}{c}\mathbf{6} \text { à } \mathbf{1 5} \text { jours } \\
\text { perdus }\end{array}$ & $\begin{array}{c}>\text { 15 jours } \\
\text { perdus }\end{array}$ \\
\hline Bio & $37 \%$ & $32 \%$ & $25 \%$ & $8 \%{ }^{*}$ \\
Conversion & $21 \%$ & $21 \%$ & $27 \%$ & $28 \%$ \\
Fruticulteurs & $11 \%$ & $29 \%$ & $25 \%$ & $32 \%$ \\
Témoin & $32 \%$ & $18 \%$ & $23 \%$ & $32 \%$ \\
\hline
\end{tabular}

* Différence significative à $95 \%$ par rapport aux autres catégories de maladies. 
Tableau 2. Caractéristiques sommaires des quatre groupes (valeurs moyennes).

Table 2. Summary characteristics of the four groups (mean values).

\begin{tabular}{|c|c|c|c|c|}
\hline & Bio & Conversion & Fruiticulteur & Témoin \\
\hline Années d'affiliation au "Meru » & $13,3[12,55 ; 14,12]$ & $1,1[0,97 ; 1,16]$ & $0[1,85 ; 3,75]$ & $0[-0,07 ; 0,20]$ \\
\hline Revenu total* & $5,411[1,149 ; 8,972]$ & $4,807[2,834 ; 6,779]$ & $6,602[3,549 ; 9,655]$ & $3,884[1,383 ; 6,385]$ \\
\hline Agriculteur & $0,8[0,648 ; 0,95]$ & $0,73[0,57 ; 0,90]$ & $0,73[0,57 ; 0,90]$ & $0,83[0,68 ; 0,97]$ \\
\hline Années de scolarisation & $3,96[2,21 ; 5,72]$ & $7,41[5,87 ; 9,16]$ & $5,41[3,49 ; 7,33]$ & $6,52[4,52 ; 8,51]$ \\
\hline Âge & $48,14[43,72 ; 51,94]$ & $42,32[37,68 ; 47,05]$ & $48,25[42,01 ; 53,99]$ & $38,21[32,35 ; 43,19]$ \\
\hline Homme & $0,53[0,34 ; 0,72]$ & $0,33[0,15 ; 0,51]$ & $0,7[0,53 ; 0,87]$ & $0,4[0,21 ; 0,59]$ \\
\hline Nombre de fils & $2,29[1,59 ; 2,98]$ & $0,83[0,39 ; 1,27]$ & $1,52[, 88 ; 2,16]$ & $1,70[1,07 ; 2,34]$ \\
\hline Personnes dans le ménage & $2,63[1,76 ; 3,50]$ & $3,45[2,73 ; 4,17]$ & $2,77[1,84 ; 3,70]$ & $3,13[2,36 ; 3,91]$ \\
\hline Autoconsommation & $5,2[4,95 ; 5,45]$ & $4,93[4,57 ; 5,30]$ & 0 & 0 \\
\hline Paiement au kilogramme & $0,83[0,69 ; 0,97]$ & $0,77[0,61 ; 0,93]$ & $0,77[0,61 ; 0,93]$ & $0,86[0,73 ; 1,00]$ \\
\hline Médecin & $0,8[0,41 ; 0,79]$ & $0,9[0,79 ; 1,01]$ & $0,86[0,74 ; 1,00]$ & $0,63[0,45 ; 0,82]$ \\
\hline Qualité nutritionnelle & $2,40[2,30 ; 2,52]$ & $2,36[2,20 ; 2,53]$ & $2,45[2,35 ; 2,55]$ & $2,25[2,20 ; 2,30]$ \\
\hline Travailler seul & $0,03[-0,03 ; 0,10]$ & $0,13[0,00 ; 0,26]$ & $0,17[0,03 ; 0,31]$ & $0,41[0,20 ; 0,61]$ \\
\hline Dispensaire & $0,13[0,00 ; 0,26]$ & $0,27[1,00 ; 0,43]$ & $0,23[0,07 ; 0,39]$ & $0,37[0,18 ; 0,55]$ \\
\hline Hôpital public & $0,87[0,74 ; 1,00]$ & $0,27[1,00 ; 0,43]$ & $0,77[0,61 ; 0,93]$ & $0,6[0,41 ; 0,79]$ \\
\hline Aucun jour perdu & $0,23[0,07 ; 0,39]$ & $0,13[0,00 ; 0,26]$ & $0,07[-0,03 ; 0,16]$ & $0,2[0,05 ; 0,35]$ \\
\hline 1 à 5 jours perdus & $0,3[0,13 ; 0,47]$ & $0,2[0,05 ; 0,35]$ & $0,27[0,1 ; 0,43]$ & $0,27[0,02 ; 0,31]$ \\
\hline 6 à 15 jours perdus & $0,4[0,21 ; 0,59]$ & $0,43[0,24 ; 0,62]$ & $0,4[0,21 ; 0,59]$ & $0,37[0,18 ; 0,55]$ \\
\hline Plus de 15 jours perdus & $0,07[0,01 ; 0,12]$ & $0,23[0,07 ; 0,39]$ & $0,27[0,1 ; 0,43]$ & $0,27[0,14 ; 0,39]$ \\
\hline Nombre d'observations & 30 & $>30$ & 30 & 30 \\
\hline
\end{tabular}

* En milliers de shillings kenyans. Un euro = 101 shillings kenyans en janvier 2005.

significative à $95 \%$. Pour la grande majorité des répondants de l'échantillon (77,7\%), l'agriculture est l'activité principale. En additionnant les revenus des activités principale et secondaire, le groupe "fruticulteurs " affiche le revenu mensuel moyen le plus élevé, suivi des agriculteurs des groupes "agriculteurs biologiques", "agriculteurs en conversion" et "groupe témoin ". Cependant, les agriculteurs du "groupe témoin" ont un âge moyen inférieur à celui des autres groupes. Pour rendre équivalents les revenus, il faut tenir compte de la composition des ménages. C'est la raison pour laquelle nous avons tenu compte du nombre d'enfants vivant dans le ménage ("nombre de fils") et du nombre total d'individus vivant dans le ménage ("personnes dans le ménage") dans le descriptif de l'échantillon. À cet égard, il est intéressant de noter que le groupe "agriculteurs biologiques" a la plus grande proportion d'enfants vivant dans la famille, ce qui signifie que notre mesure du revenu total est en quelque sorte biaisée par le haut pour ce groupe. Néanmoins, le groupe " agriculteurs biologiques " affiche également la part d'autoconsommation la plus élevée.

En ce qui concerne le sexe des répondants, la part d'hommes dans le groupe "agriculteurs biologiques" reste dans la moyenne par rapport aux autres groupes. En termes d'éducation, les agriculteurs du groupe "agriculteurs biologiques " affichent le plus faible nombre d'années de scolarisation. Ce résultat s'explique par deux facteurs. D'abord, comme on le constate souvent dans les pays en voie de développement, il existe une corrélation entre l'âge et les années de scolarisation, due aux effets progressifs des politiques d'éducation dans le temps. En outre, la coopérative a débuté avec les agriculteurs les plus pauvres, dont la seule activité économique était l'agriculture. Par conséquent, l'affiliation à l'agriculture biologique est corrélée avec un plus faible niveau de scolarisation. Nous avons également pris en compte les variables disponibles liées aux conditions et aux pratiques sanitaires. Celles-ci comprennent la visite aux dispensaires ("dispensaire") plutôt qu'aux hôpitaux ("hôpital public") en cas de maladie et une variable muette ("médecin ") qui renvoie à la présence d'un médecin pendant le dernier accouchement. En général, les agriculteurs du groupe " agriculteurs biologiques " ont davantage recours aux hôpitaux publics (par rapport aux dispensaires) que les autres groupes, mais ils n'affi- chent pas de préférence quant aux soins médicaux.

Une autre mesure de bien-être qui a un impact direct sur la santé dans les pays pauvres est la nutrition. Dans notre enquête, nous disposons d'informations concernant la fréquence à laquelle sont consommées certaines denrées alimentaires (ugali, chapati, riz, maïs, fèves, œufs, lait, poulet, autres viandes, poisson, pommes de terre, légumes verts, fruits frais). À partir de cette base, nous construisons un indice de qualité nutritionnelle en attribuant des valeurs décroissantes (d'un maximum de 5 à un minimum d'1) aux modalités de fréquence susmentionnées. Enfin, nous calculons notre indice synthétique comme la moyenne des valeurs attribuées à chaque bien alimentaire. L'indice de qualité nutritionnelle semble être relativement homogène entre les quatre groupes, bien qu'il soit plus faible dans le groupe témoin.

Bien évidemment, l'état de santé dépend de différentes caractéristiques liées aux conditions de travail, car les individus en âge de travailler passent une grande partie de leur temps sur le lieu de travail. À cet égard, nous examinons deux variables : - une variable muette qui prend une valeur 1 dans le cas d'une rémunération fondée 
sur la quantité produite ("paiement au kilo ") et 0 dans le cas d'un salaire fixe ;

- une variable muette qui prend une valeur 1 si l'agriculteur travaille seul ("travailler seul") plutôt que dans un groupe agriculteurs.

Il convient de noter que les agriculteurs du groupe "agriculteurs biologiques" sont les moins présents parmi ceux qui travaillent seuls. Ceci signifie que la corrélation négative entre le nombre de jours de travail perdus pour cause de maladie et l'affiliation au commerce équitable ne peut pas être expliquée par le fait que les travailleurs agriculteurs biologiques ne peuvent pas se permettre d'être absents.

En ce qui concerne nos mesures de l'état de santé, le tableau 2 montre que le groupe " agriculteurs biologiques " affiche le nombre le plus élevé de travailleurs appartenant à la catégorie de travailleurs ayant perdu le moins de jours de travail, ainsi que le nombre le plus faible de travailleurs appartenant à la catégorie de travailleurs ayant perdu le plus de jours de travail pour cause de maladie. Ces résultats confirment les résultats présentés dans le tableau 1. Il faut noter que nous obtenons une significativité statistique de $95 \%$ lorsque nous comparons le groupe "agriculteurs biologiques" et le "groupe témoin "sur la base du nombre de travailleurs ayant perdu plus de 15 jours de travail pour cause de maladie. Cependant il ne faut pas oublier que les trois autres catégories de jours de travail perdus sont grandes et ont donc tendance à réduire les différences entre les groupes.

Enfin, les estimations économétriques montrent que le nombre d'années d'affiliation au commerce équitable réduit de façon significative le nombre de jours perdus pour cause de maladie, net de l'effet des variables de contrôle traditionnelles (revenu, richesse, âge, éducation, qualité nutritionnelle) proposées par la littérature et également après avoir contrôlé pour les effets dus au biais de sélection ${ }^{2}$.

\section{Conclusion}

L'analyse descriptive souligne une relation entre l'appartenance au groupe "agriculteurs biologiques" et l'état de santé mesuré par le nombre de jours de travail perdus pour cause de maladie. Cette relation ne peut s'expliquer par des différences de revenus ou de scolarisation entre les différents groupes. Les estimations économétriques confirment la significativité de ce résultat.

Ce résultat est surprenant, non seulement parce que les conditions socioéconomiques générales sont homogènes entre les quatre groupes, mais aussi parce que les agriculteurs affiliés sont beaucoup plus âgés et moins instruits que ceux du groupe témoin. Cela signifie que les facteurs traditionnellement perçus comme des déterminants de l'état de santé dans les pays pauvres ne jouent aucun rôle pour expliquer les différences d'état de santé dans notre échantillon. Nos résultats éclairent de manière significative la question de l'impact du commerce équitable sur le bien-être et la capacité productive des producteurs. Au-delà d'un effet sur la productivité que font ressortir certaines études d'impact, l'affiliation au commerce équitable produit un autre effet indirect positif sur la création de valeur en agissant sur l'état de santé des producteurs et sur le nombre de jours travaillés.

En examinant les autres caractéristiques de l'affiliation au commerce équitable présentées dans l'introduction, nous mettons en avant la possibilité d'un cercle vertueux basé sur les échanges marchands, dans lequel l'alliance entre des consommateurs socialement responsables, des importateurs et détaillants du commerce équitable et des producteurs marginalisés crée les prémisses d'une amélioration en termes de capital humain, d'accès au marché et de productivité, amélioration nécessaire pour promouvoir l'intégration et sortir ces producteurs de la trappe de la pauvreté.

\section{Références}

Bacon C. Confronting the Coffee Crisis : Can Fair Trade, Organic, and Specialty Coffees Reduce Small-Scale Farmer Vulnerability in Northern Nicaragua? World Dev 2005; 33 : 497-511.

Becchetti L, Costantino M. Fair Trade on marginalized producers: an impact analysis on Kenyan farmers. World Dev 2008 ; 36 : 823-42.
Becchetti L, Costantino M, Portale E. Human capital, externalities and tourism: three unexplored sides of the impact of FT affiliation on primary producers. CEIS Working Paper, $\mathrm{n}^{\circ}$ 262. Rome : Université de Tor Vergata, 2007.

Becchetti L, Rosati F. Globalisation and the death of distance in social preferences and inequity aversion: empirical evidence from a pilot study on fair trade consumers. World Econ 2007 ; 30 : 807-30.

Clark A, Frijters P, Shields M. Income and Happiness: Evidence, Explanations and Economic Implications., Document de travail No. 2006-24. Paris : Jourdan sciences économiques, 2006.

Deaton A. Global Patterns of Income and Health: Facts, Interpretations and Policies. NBER Document de travail, $\mathrm{n}^{\circ} 12$ 735. Cambridge (Massachussets) : NBER, 2006.

Frey B, Stutzer A. Happiness and Economics. How the economy and institutions affect wellbeing. Princeton: Princeton University Press, 2002.

Frey B, Stutzer A. What Can Economists Learn from Happiness Research? J Econ Lit 2002; $40: 402-35$.

Hayes, M. Strategic management implication of the ethical consumer, 2004. <http://www. fairtraderesearch.org>

Maseland R, De Vaal A. How Fair is Fair Trade? De Economist $2002 ; 150: 251-72$.

Moore G. The Fair Trade Movement : parameters, issues and future research. J Bus Ethics $2004 ; 53: 73-86$

Pariente, W. The impact of fair trade on a coffee cooperative in Costa Rica. Aproducers behaviour approach. Paris : université Paris I Panthéon Sorbonne, 2000.

Redfern A, Snedker P. Creating market opportunities for small enterprises: experiences of the fair trade movement. Genève : OIT, 2002.

Strauss J, Thomas D. Health, Nutrition and Economic Development. J Econ Lit 1998; 36 : 766-817.

Trewavas AT. A critical assessment of organic farming-and-food assertions with particular respect to the UK and the potential environmental benefits of no-till agriculture. Crop Prot $2004 ; 23$ : 757-81.

Williams CM. Nutritional quality of organic food : shades of grey or shades of green? Proc Nutr Soc 2002; 61 : 19-24.

Worthington V. Nutritional Quality of Organic versus Conventional Fruits, Vegetables and Grains. JAltern Complem Med 2001; 7 : 161-73.

$\overline{2}$ Les résultats sont disponibles sur demande. 